\title{
Biomimetic Synthesis of Zinc Oxide 3D Architectures with Gelatin as Matrix
}

\author{
Yong Gan, Fubo Gu, Dongmei Han, Zhihua Wang, and Guangsheng Guo \\ State Key Laboratory of Chemical Resource Engineering, Beijing University of Chemical Technology, Beijing 100029, China
}

Correspondence should be addressed to Guangsheng Guo, guogs@mail.buct.edu.cn

Received 27 November 2009; Accepted 31 March 2010

Academic Editor: Steve Acquah

Copyright () 2010 Yong Gan et al. This is an open access article distributed under the Creative Commons Attribution License, which permits unrestricted use, distribution, and reproduction in any medium, provided the original work is properly cited.

Peanut-like and flower-like zinc oxide 3D architectures were synthesized via a facile biomimetic process using gelatin as matrix. Techniques of XRD, SEM, HRTEM, FT-IR, and UV-vis absorption spectra were used to characterize the structure and property of the zinc oxide architectures. The experimental results show that the peanut-like $\mathrm{ZnO}$ and flower-like $\mathrm{ZnO}$ architectures can be obtained through changing the $\mathrm{Zn}^{2+}$ concentration or the aging time. FT-IR spectra indicate that the $\mathrm{Zn}^{2+}$ is coupled with the $\mathrm{C}=\mathrm{O}$ bond of the gelatin molecules through the electrostatic interaction. Based on the experimental process, the possible growth mechanism of the $\mathrm{ZnO} 3 \mathrm{D}$ architectures is proposed. UV-vis absorption spectrum of the peanut-like $\mathrm{ZnO}$ has a broad absorption band in the UV region, and the blue-shifting of the band is observed.

\section{Introduction}

Nanomaterials have attracted much attention because they have special characteristics in optical, photovoltaic, and catalytic applications that differ from those of bulk materials [1]. Zinc oxide has a wide band gap $(3.37 \mathrm{eV})$ and a large exciton binding energy $(60 \mathrm{meV})$ at room temperature and has been extensively investigated for applications in luminescence, photocatalysts, surface acousite wave filters, piezoelectric transducers and actuators, gas sensors, solar cells and so on [2-8]. Recently, many synthesis methods have been employed for the growth of $\mathrm{ZnO}$ nanomaterials such as the hydrothermal process [9-11], the conventional sputter deposition technique [12], the chemical vapor deposition (CVD) [13-15] and the thermal evaporation [4]. Moreover, many graceful nano-architectures have been prepared by different techniques, such as nanowires, nanobridges, nanosnails, nanobundles and nanocombs $[10,15,16]$. However, the methods used to prepare these types of $\mathrm{ZnO}$ usually require relatively high temperature and sometimes complicated process. Comparatively, the wet chemical method, for it employs a low growth temperature $\left(<100^{\circ} \mathrm{C}\right)$ process and has good potential for scale-up, becomes one of the successful techniques for preparing nanosized $\mathrm{ZnO}$ [17-22].
Biominerals are usually formed on the surface of organic templates (or matrixes). Recently, inorganic materials with higher performance and more exquisite morphologies have been obtained by biomimetic synthesis. Simulating the biomineralization process of the nucleation and growth of inorganic materials mediated by organic matrixes has become the focus of material science $[23,24]$. Gelatin is the denaturation product of collagen which is the major structural protein in the connective tissue of animal skin and bones. Gelatin has been investigated by in vitro biomineralization [25-31]. Heterogeneous nucleation of $\mathrm{ZnO}$ using gelatin as the organic matrix has been studied [20]. However, the reported synthetic methods are limited to the growth of $1 \mathrm{D}$ or $2 \mathrm{D} \mathrm{ZnO}$ (thin films) and only few on 3D ordered architectures [6]. In the present work, a simple and biomimetic process was employed to synthesize 3D zinc oxide architectures at room temperature. The biomimetic growth and self-assembly of zinc oxide 3D architectures in the gelatin solution were investigated, and the formation mechanism of the 3D $\mathrm{ZnO}$ architectures was also discussed.

\section{Experimental Section}

2.1. Materials. Gelatin was obtained from Beijing Aoboxing Universeen Bio-Tech Co. Ltd (Beijing, China). 
$\mathrm{Zn}\left(\mathrm{NO}_{3}\right)_{2} \cdot 6 \mathrm{H}_{2} \mathrm{O}$, absolute ethyl alcohol and ammonia were purchased from Beijing Chemical Reagent Co. Ltd (Beijing, China) and all of reagents were of analytical grade. De-ionized water was used in each synthesis and washing step.

2.2. Synthesis of Zinc Oxide 3D Architectures. In this experiment, a simple biomineralized method to obtain zinc oxide crystals was adopted. In brief, the aqueous solution of gelatin as mineralized solution was prepared using 3 g gelatin dissolved in $100 \mathrm{ml}$ de-ionized water. The concentration of the gelatin was $3 \%(\mathrm{wt}) .1 .84 \mathrm{~g} \mathrm{Zn}\left(\mathrm{NO}_{3}\right)_{2} \cdot 6 \mathrm{H}_{2} \mathrm{O}$ was added in $100 \mathrm{ml}$ as-prepared gelatin solution and dissolved. The mixed solution was stirred continuously and titrated with ammonia $(6 \mathrm{~mol} / \mathrm{L})$ at room temperature, and then the $\mathrm{pH}$ value of the mixed solution was adjusted to 8.0. After being stirred for 30 minutes, the suspension was kept at $30^{\circ} \mathrm{C}$ for 24 hours. The precipitate was obtained by centrifugation at a speed of $4500 \mathrm{rpm}$ and then washed thoroughly with de-ionized water to remove the diffluent salts. Three cycles of washing and centrifuging were required. Afterwards, the precipitate was washed twice with absolute ethyl alcohol. Finally, the sample was harvested by centrifugation and oven-dried at $60^{\circ} \mathrm{C}$ for 12 hours. According to the same process, $2.97 \mathrm{~g}$ $\mathrm{Zn}\left(\mathrm{NO}_{3}\right)_{2} \cdot 6 \mathrm{H}_{2} \mathrm{O}$ was added in gelatin solution and the other sample was obtained.

2.3. Characterization. The crystallization and purity of the samples were characterized by X-ray diffraction (XRD) analysis with a D/max 2500VB2+/PC X-ray diffractometer using graphite monochromatized $\mathrm{Cu} K \alpha$ radiation $(\lambda=$ $0.15406 \mathrm{~nm})$ at a scanning speed of $10 \% \mathrm{~min}^{-1}$. Field emission scanning electron microscopy (FESEM, Hitachi S-4700) was employed to characterize the morphological features of the samples that were sputter-coated with gold prior to examination. The detailed morphology and structural characterization were investigated through high-resolution transmission electron microscope (HRTEM, JEM3010). For infrared adsorption analysis, $1 \mathrm{mg}$ of the samples was carefully mixed with $200 \mathrm{mg}$ of $\mathrm{KBr}$ (infrared grade) and pelletized (diameter of the disk shaped pellet $=7 \mathrm{~mm}$ ) under the pressure of 4 tons for 1 minute. The pellets were analyzed by using a Nicolet Avatar 370 MCT Fouriertransform infrared (FT-IR) spectrometer in the range of $4000-400 \mathrm{~cm}^{-1}$. The optical property of the samples was measured by UV-Vis spectrophotometer (Hitachi Model U3010).

\section{Results and Discussions}

3.1. The Structural Characterization of $\mathrm{ZnO}$ Nanoarchitectures. Figure 1 shows the XRD patterns of the products. Product A and Product B were obtained when the concentrations of $\mathrm{Zn}^{2+}$ were $0.06 \mathrm{~mol} / \mathrm{L}$ and $0.1 \mathrm{~mol} / \mathrm{L}$, respectively. The diffraction peaks were indexed as the (100), (002), (101), (102), (110), (103), (200), (112), (201), (004), (202), (104), and (203) reflections of wurtzite (hexagonal) structured $\mathrm{ZnO}$ (JCPDS card No. 65-3411, lattice parameters of $\mathrm{a}=\mathrm{b}=$

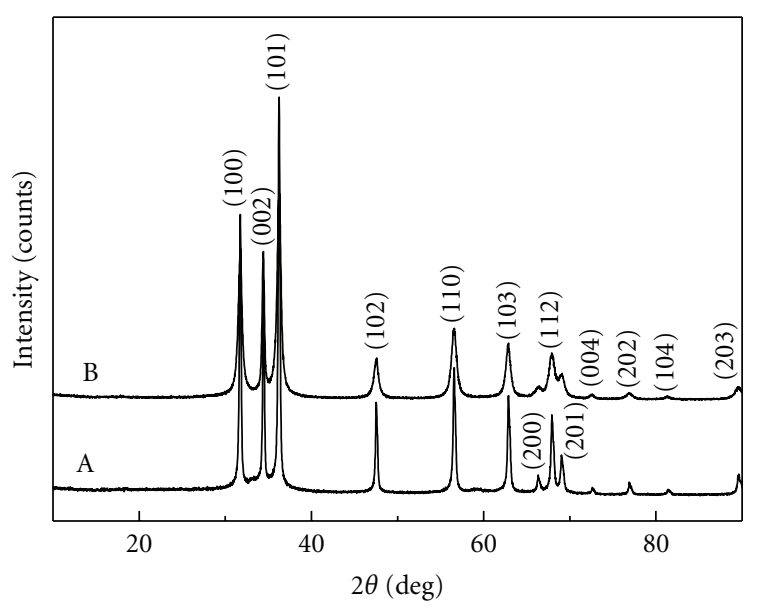

FIGURE 1: XRD patterns of zinc oxide synthesized at the different $\mathrm{Zn}^{2+}$ concentration in $3 \%(\mathrm{wt})$ gelatin solution: $\mathrm{A}, 0.06 \mathrm{~mol} / \mathrm{L}$ $\mathrm{Zn}\left(\mathrm{NO}_{3}\right)_{2} \cdot 6 \mathrm{H}_{2} \mathrm{O} ; \mathrm{B}, 0.1 \mathrm{~mol} / \mathrm{L} \mathrm{Zn}\left(\mathrm{NO}_{3}\right)_{2} \cdot 6 \mathrm{H}_{2} \mathrm{O}$.

$3.25 \AA, c=5.207 \AA)$. The products are well crystallized and the crystallization degree of Product $A$ is better than that of Product $B$ according to the intensity of the XRD pattern. No peaks associated with other crystalline forms are detected, which indicates that $\mathrm{ZnO}$ crystals were successfully synthesized through the simple biomimetic process.

3.2. The Morphology of the $3 \mathrm{D} \mathrm{ZnO}$ Architectures. Figure 2 presents the FESEM images of the $3 \mathrm{D} \mathrm{ZnO}$ architectures. It can be seen that the as-prepared products are mainly composed of two types of architectures: peanut-like (Figure 2(a)) and flower-like (Figure 2(b)) structures. The peanut-like products are obtained when the concentration of $\mathrm{Zn}^{2+}$ is $0.06 \mathrm{~mol} / \mathrm{L}$, and the morphologies of the $3 \mathrm{D}$ peanut-like $\mathrm{ZnO}$ architectures are with length ranging from $500 \mathrm{~nm}$ to $1 \mu \mathrm{m}$ and width varying from $200 \mathrm{~nm}$ to $300 \mathrm{~nm}$. However, as the concentration of $\mathrm{Zn}^{2+}$ ions increases to $0.1 \mathrm{~mol} / \mathrm{L}$, flower-like $\mathrm{ZnO}$ structures produce, consisting of nano-rods with $80 \mathrm{~nm}$ in length and $20 \mathrm{~nm}$ in diameter. These experimental results reveal that the development of $\mathrm{ZnO}$ architectures in gelatin solution is greatly influenced by the concentration ratio of $\mathrm{Zn}^{2+}$ to gelatin.

High-Resolution Transmission electron microscopy (HRTEM) studies were carried out to examine the crystallography and structure of the peanut-like $\mathrm{ZnO}$ architectures. Figure 3 shows the HRTEM images of the peanut-like $\mathrm{ZnO}$ sample. Figure $3(\mathrm{a})$ is the low-magnification TEM image of the peanut-like $\mathrm{ZnO}$ after sonication treatment in ethanol for 30 minutes. The dimensions of the peanut-like $\mathrm{ZnO}$ are about $500-800 \mathrm{~nm}$ in length and about $200 \mathrm{~nm}$ in width, which are consistent in the FESEM observations. The HRTEM images in Figure 3(b) show that the peanut-like $\mathrm{ZnO}$ architectures are organized with nanorods, and have a clean and perfect lattice structure without dislocation and stacking faults. The lattice space of adjacent planes is about $0.25 \mathrm{~nm}$, corresponding to the (101) fringe perpendicular to the growth direction, which is consistent with that of the bulk wurtzite $\mathrm{ZnO}$ crystal. 


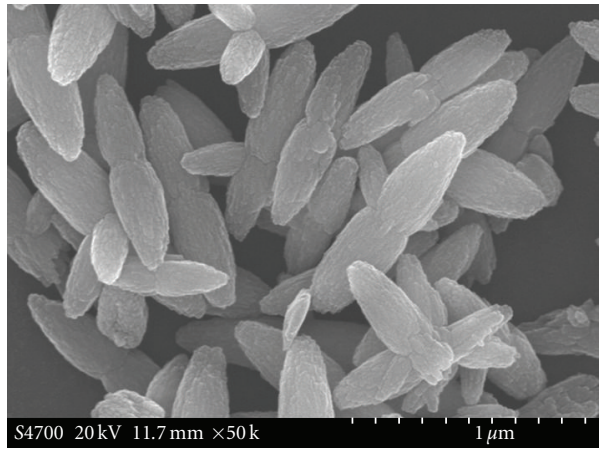

(a)

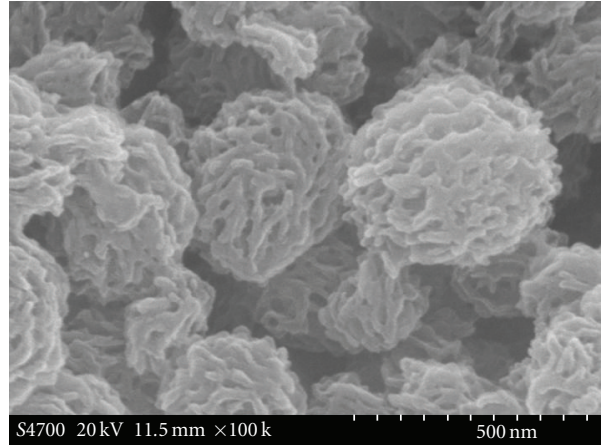

(b)

FIGURE 2: FESEM images of the zinc oxide synthesized at the different $\mathrm{Zn}^{2+}$ concentration in $3 \%$ (wt) gelatin solution: (a), $0.06 \mathrm{~mol} / \mathrm{L}$; (b), $0.1 \mathrm{~mol} / \mathrm{L}$.

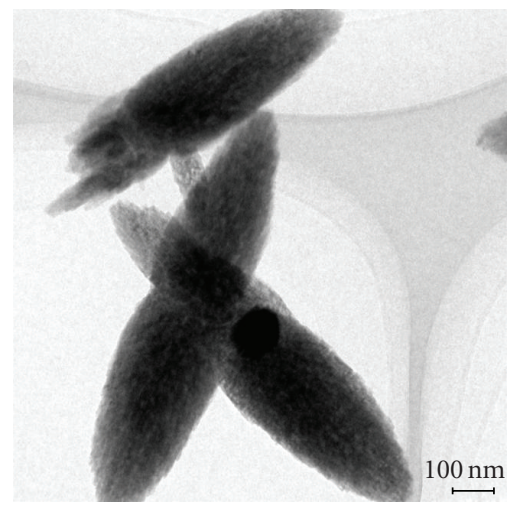

(a)

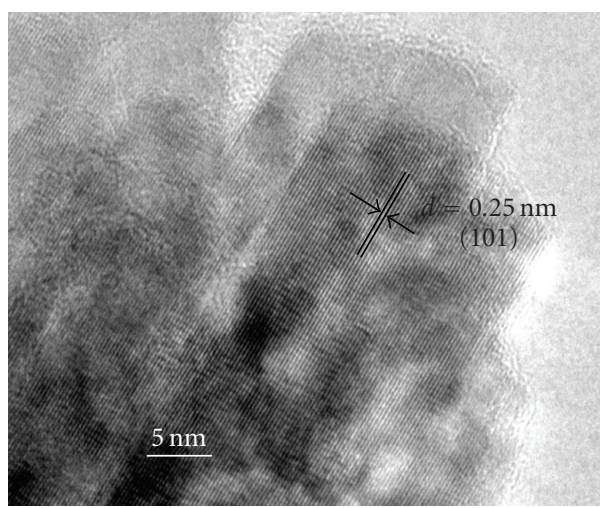

(b)

FIGURE 3: HRTEM images of the peanut-like ZnO: (a), low-magnification; (b), high-magnification.

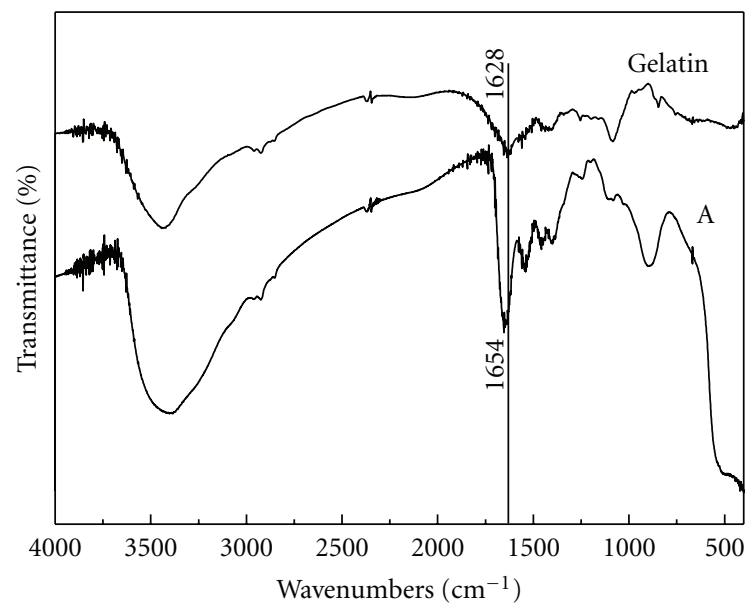

FIGURE 4: FT-IR spectra of the peanut-like $\mathrm{ZnO}$ and the gelatin.

3.3. FTIR Measurement. Figure 4 is the FT-IR spectra of the gelatin and the peanut-like $\mathrm{ZnO}$. It shows that the characteristic absorption bands at 1260, 1416 and $1628 \mathrm{~cm}^{-1}$ are assigned to the amide I, II, III of gelatin [27]. The $1342 \mathrm{~cm}^{-1}$ band in gelatin represents the carboxyl group, which is attributed to the so-called wagging vibration of praline side chains. These absorption bands indicate that a small amount of gelatin exists in the $\mathrm{ZnO}$ architectures. Compared with the spectra, the reflectance strong $\mathrm{C}=\mathrm{O}$ peak at $1628 \mathrm{~cm}^{-1}$ in gelatin shifts to higher wavenumber at $1654 \mathrm{~cm}^{-1}$ in the $\mathrm{ZnO}$ sample due to the $\mathrm{Zn}^{2+}$ ions coupled with the $\mathrm{C}=\mathrm{O}$ through electrostatic interaction. This result suggests that the nucleation and self-organization of $\mathrm{ZnO}$ nanocrystals can be controlled by the gelatin macromolecules, and the carbonyl groups on the surface of gelatin molecules can be the nucleation sites of $\mathrm{ZnO}$. Then, the $\mathrm{ZnO}$ nanocrystals precipitate on the surface of the gelatin macromolecules and are spontaneously formed into $3 \mathrm{D} \mathrm{ZnO}$ architectures.

3.4. The Proposed Growth Model and Mechanism. To further investigate the growth process of the peanut-like $\mathrm{ZnO}$ architectures, FESEM studies were carried out for the products obtained at different reaction times. Figure 5 shows the FESEM images of the product obtained at the aging time of 10 minutes, 30 minutes, 1 hour, 2 hours, 6 hours, 12 hours, 18 hours and 24 hours. It can be found that the morphology of as-synthesized samples vary from nanoparticle and flowerlike structure to peanut-like structure. When the aging time is less than 2 hours, the particle structures produce, as 


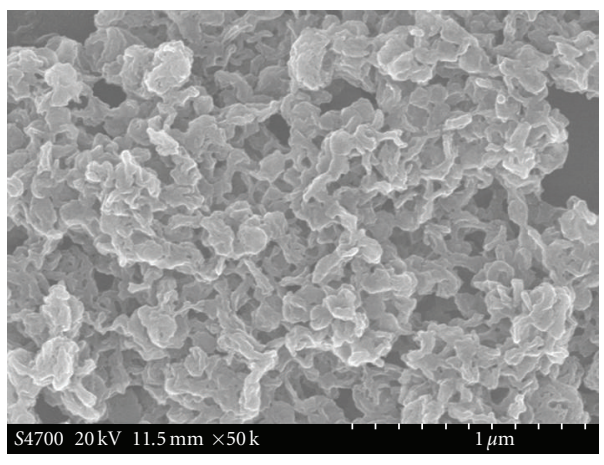

(a)

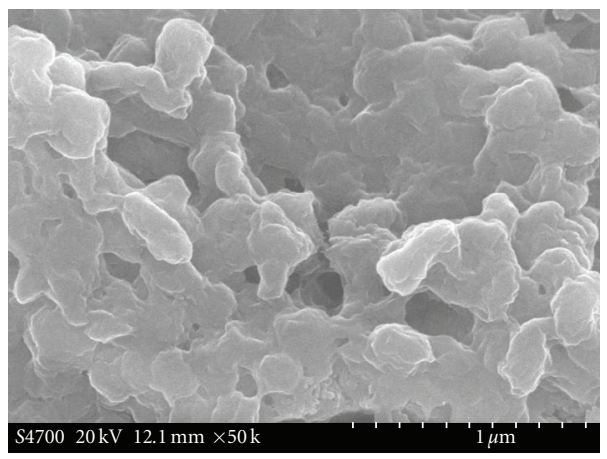

(c)

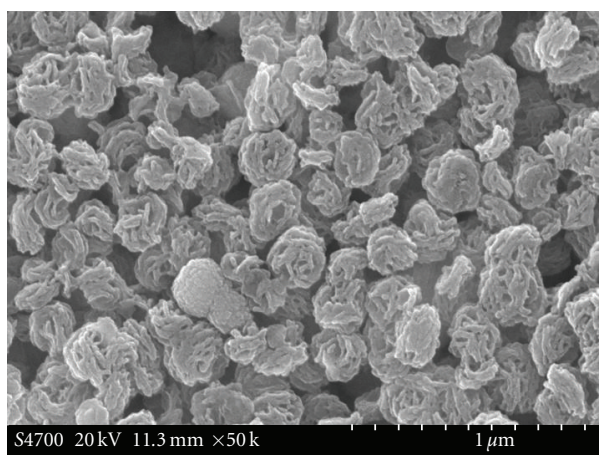

(e)

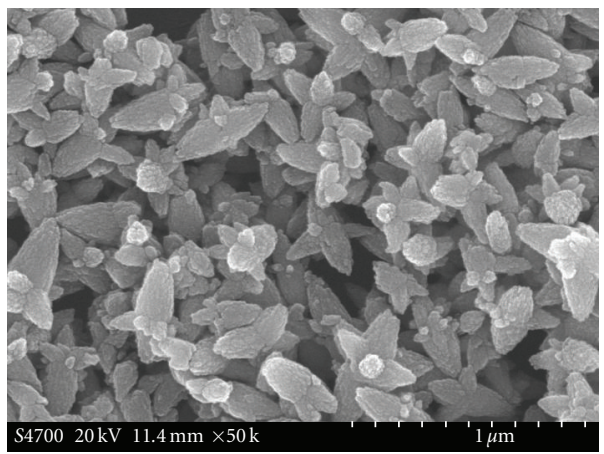

(g)

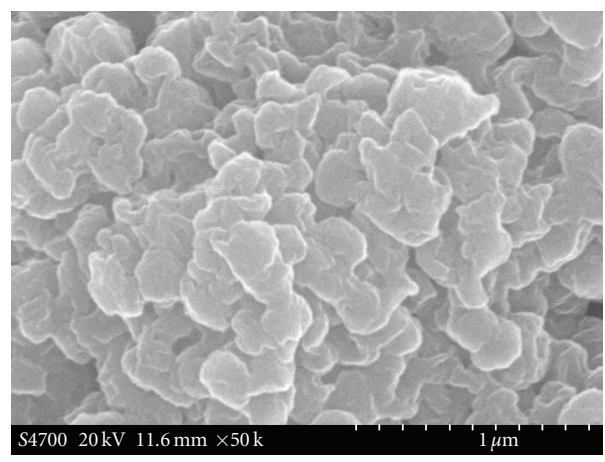

(b)

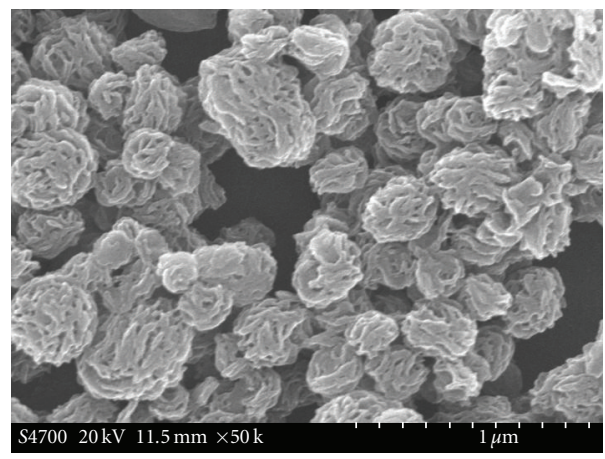

(d)

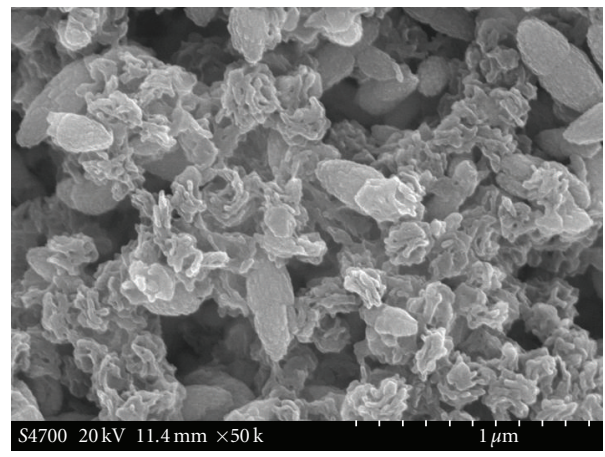

(f)

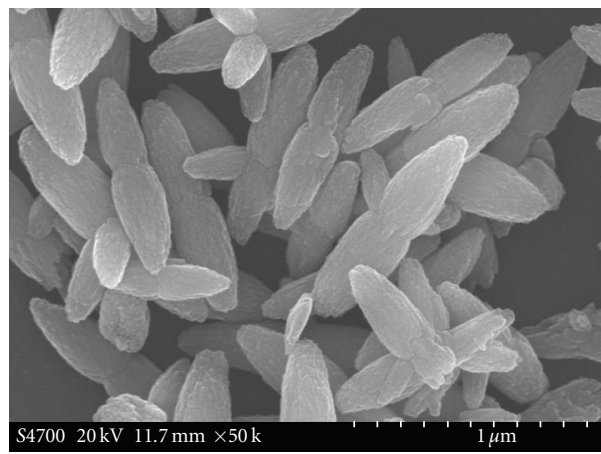

(h)

FIGURE 5: FESEM images of the samples synthesized at different reaction times: (a) 10 minutes; (b) 30 minutes; (c) 1 hour; (d) 2 hours; (e) 6 hours; (f) 12 hours; (g) 18 hours; (h) 24 hours. 


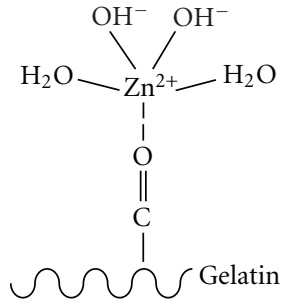

(a)

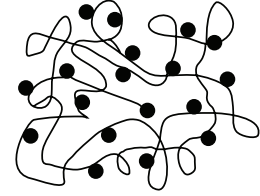

(b)

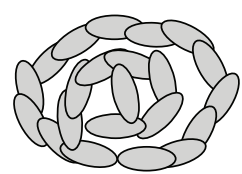

(c)

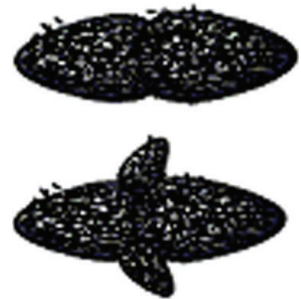

(d)

Scheme 1: Illustration of growth process of 3D ZnO architectures: (a) nucleation; (b) growth; (c) flower-like structure; (d) peanut-like structure.

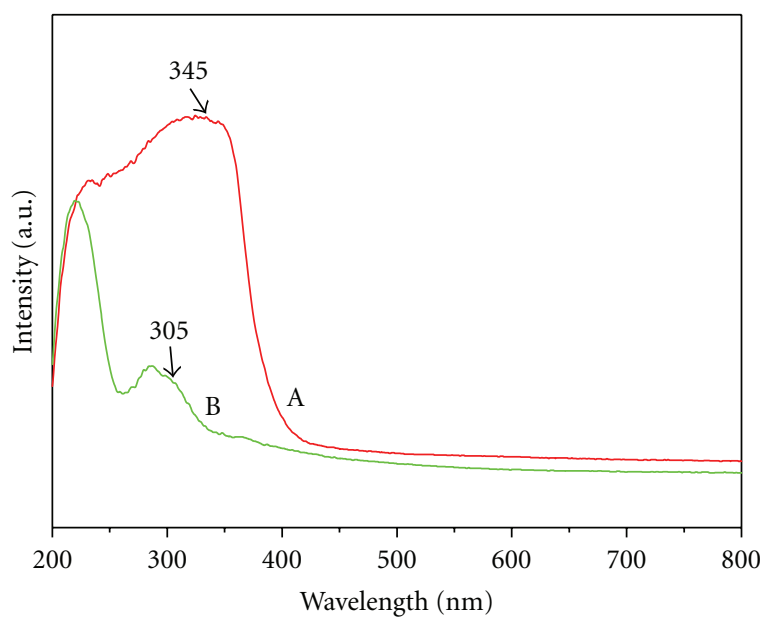

Figure 6: UV-vis spectra of the $\mathrm{ZnO}$ samples: Curve $\mathrm{A}$ is the peanut-like $\mathrm{ZnO}$; Curve $\mathrm{B}$ is the flower-like $\mathrm{ZnO}$.

shown in Figures 5(a)-5(c). In comparison, as the aging time reaches 2 hours and 6 hours, the flower-like structures form (Figures 5(d)-5(f)). The peanut-like structures are shown in Figure 5(e) and the quantity of the flower-like structures decreases when the aging time is more than 12 hours. As the time comes up to 18 hours and 24 hours, lots of peanut-like architectures produce (Figure $5(\mathrm{~g})$ ). In the growth process, the gelatin molecules can induce the nanoparticles to become flower-like and peanut-like 3D architectures. The formation of $3 \mathrm{D} \mathrm{ZnO}$ architectures could be a self-assembly process. Individual $\mathrm{ZnO}$ nanoparticles self-assemble to hierarchical nanostructures as the primary unit, and then hierarchical nanostructures interact via Van der Waals forces and electrostatic forces, which results in their spontaneous self-organization and the formation of the higher-order structures [12].

Schematic representation of nucleation, growth and selfassembled aggregation of the $3 \mathrm{D} \mathrm{ZnO}$ architectures are displayed in Scheme 1 based on the process of growth at different aging times. The nucleation begins with the electrostatic attraction between the positive charges of metallic ions and the negative charges of carboxylic groups of the gelatin molecule (Scheme 1(a)) [20]. The gelatin mainly disperses as random coils (Scheme 1(b)) and these random coils behave as organic matrixes holding $\mathrm{Zn}^{2+}$ cations on the negative charge of gelatin molecules, and the nucleation can be initiated from these active sites as a heterogeneous reaction. After nucleation, $\mathrm{ZnO}$ growth takes place in the free space among gelatin macromolecule chains with obvious consequences on the morphology of the inorganic deposits. Owing to the effect of the Van der Waals forces, electrostatic forces and hydrogen bonds among the gelatin macromolecules chains, the $\mathrm{ZnO}$ nanocrystals as primary units self-organize to the flower-like 3D structures (Scheme 1(c)). According to the $\mathrm{XRD}$ results, the crystallization degree of the flower-like $\mathrm{ZnO}$ is weaker. As the mineralization is in progress, the $\mathrm{ZnO}$ crystals would recrystallize further and the single flower-like 3D architecture can be transformed and organized to the peanut-like 3D architectures as a sub-unit structure of selfassembly process (Scheme $1(\mathrm{~d})$ ). The HRTEM results show that the peanut-like architectures, which are composed of rod-like $\mathrm{ZnO}$, are better crystallization and stabler morphology to wurtzite $\mathrm{ZnO}$ [30]. Therefore, when the reaction time is more than 18 hours, the final particle structures incline to turn into $3 \mathrm{D}$ peanut-like $\mathrm{ZnO}$ architectures.

3.5. UV-Vis Characterization. Figure 6 presents the UV-Vis absorption spectra of the $\mathrm{ZnO}$ samples. Curve $\mathrm{A}$ is the sample of peanut-like $\mathrm{ZnO}$ and Curve $\mathrm{B}$ is the sample of flower-like $\mathrm{ZnO}$. It can be seen from Curve A in Figure 6 that there is intensive absorption in the ultraviolet band of about $200-400 \mathrm{~nm}$. With respect to the bulk absorption edge appearing at $373 \mathrm{~nm}$ at room temperature, a stronger excitonic absorption feature at about $345 \mathrm{~nm}$ is blue shifted about $30 \mathrm{~nm}[1,32]$. The fact that the excitonic peak shifts to blue results from their decreasing crystal size due to the pronounced quantum confinement effect in the $\mathrm{ZnO}$ nanocrystallites [32]. As to Curve B, on the other hand, the absorption peak is located at $305 \mathrm{~nm}$ and shows a poor UV absorption because of weaker crystallization.

\section{Conclusions}

In summary, zinc oxide 3D architectures were synthesized by the biomimetic process in the gelatin solution at the room temperature. XRD, FESEM, HRTEM, FT-IR and UVVis absorption spectra had been used to characterize the as-obtained $\mathrm{ZnO}$ samples. The XRD patterns prove that as-prepared products indexed to the wurtzite (hexagonal) structure of $\mathrm{ZnO}$ are well crystallized and have no obvious 
impurity phase. By changing concentrations of $\mathrm{Zn}^{2+}$ ions or the reaction time, peanut-like $3 \mathrm{D} \mathrm{ZnO}$ architectures and flower-like 3D $\mathrm{ZnO}$ architectures can be successfully synthesized. FT-IR spectra show that the $\mathrm{Zn}^{2+}$ ions are coupled with the $\mathrm{C}=\mathrm{O}$ bond through electrostatic interaction, which suggests that the amide groups and carboxyl groups on the surface of gelatin molecules are the nucleation sites of $\mathrm{ZnO}$. The $\mathrm{ZnO}$ nanocrystals then precipitate on the surface of the gelatin and spontaneously self-organize to $3 \mathrm{D} \mathrm{ZnO}$ architectures. The UV-Vis absorption spectra of the peanutlike $\mathrm{ZnO}$ nanocrystals reveal a stronger excitonic absorption feature at about $345 \mathrm{~nm}$. It is expected that this study can offer a convenient and efficient route for the preparation of $\mathrm{ZnO}$ architectures.

\section{Acknowledgments}

This work was supported by the China National Natural Science Fund (No. 20875007) and Beijing Municipal Science and Technology Development project (No. Z080003032208012).

\section{References}

[1] C. He, T. Sasaki, Y. Shimizu, and N. Koshizaki, "Synthesis of $\mathrm{ZnO}$ nanoparticles using nanosecond pulsed laser ablation in aqueous media and their self-assembly towards spindle-like ZnO aggregates," Applied Surface Science, vol. 254, no. 7, pp. 2196-2202, 2008.

[2] K. M. Sulieman, X. Huang, J. Liu, and M. Tang, "Formation of $\mathrm{ZnO}$ three-side teethed nanostructures," Materials Letters, vol. 61, no. 8-9, pp. 1756-1759, 2007.

[3] X. An, C. Cao, and H. Zhu, "Bio-inspired fabrication of $\mathrm{ZnO}$ nanorod arrays and their optical and photoresponse properties," Journal of Crystal Growth, vol. 308, no. 2, pp. 340347, 2007.

[4] C. Li, G. Fang, N. Liu, Y. Ren, H. Huang, and X. Zhao, "Snowflake-like $\mathrm{ZnO}$ structures: self-assembled growth and characterization," Materials Letters, vol. 62, no. 12-13, pp. 1761-1764, 2008.

[5] L. Wu, Y. Wu, and Y. Lü, "Self-assembly of small $\mathrm{ZnO}$ nanoparticles toward flake-like single crystals," Materials Research Bulletin, vol. 41, no. 1, pp. 128-133, 2006.

[6] J. Liu, X. Huang, Y. Li, J. Duan, and H. Ai, "Large-scale synthesis of flower-like $\mathrm{ZnO}$ structures by a surfactantfree and low-temperature process," Materials Chemistry and Physics, vol. 98, no. 2-3, pp. 523-527, 2006.

[7] X. Cao, X. Lan, C. Zhao, W. Shen, and D. Yao, "Porous $\mathrm{ZnS} / \mathrm{ZnO}$ microspheres prepared through the spontaneous organization of nanoparticles and their application as supports of holding CdTe quantum dots," Materials Research Bulletin, vol. 43, no. 5, pp. 1135-1144, 2008.

[8] Z. Jia, L. Yue, Y. Zheng, and Z. Xu, "Rod-like zinc oxide constructed by nanoparticles: synthesis, characterization and optical properties," Materials Chemistry and Physics, vol. 107, no. 1, pp. 137-141, 2008.

[9] Y. Ni, G. Wu, X. Zhang, et al., "Hydrothermal preparation, characterization and property research of flowerlike $\mathrm{ZnO}$ nanocrystals built up by nanoflakes," Materials Research Bulletin, vol. 43, no. 11, pp. 2919-2928, 2008.
[10] Z. Fang, K. Tang, G. Shen, D. Chen, R. Kong, and S. Lei, "Self-assembled $\mathrm{ZnO} 3 \mathrm{D}$ flowerlike nanostructures," Materials Letters, vol. 60, no. 20, pp. 2530-2533, 2006.

[11] T. Ghoshal, S. Kar, J. Ghatak, and S. Chaudhuri, " $\mathrm{ZnO}$ nanocones: solvothermal synthesis and photoluminescence properties," Materials Research Bulletin, vol. 43, no. 8-9, pp. 2228-2238, 2008.

[12] S. C. Lyu, Y. Zhang, H. Ruh, et al., "Low temperature growth and photoluminescence of well-aligned zinc oxide nanowires," Chemical Physics Letters, vol. 363, no. 1-2, pp. 134-138, 2002.

[13] J.-J. Wu and S.-C. Liu, "Catalyst-free growth and characterization of ZnO nanorods," Journal of Physical Chemistry B, vol. 106, no. 37, pp. 9546-9551, 2002.

[14] M. C. Newton and P. A. Warburton, "ZnO tetrapod nanocrystals," Materials Today, vol. 10, no. 5, pp. 50-54, 2007.

[15] M. Muruganandham and J. J. Wu, "Synthesis, characterization and catalytic activity of easily recyclable zinc oxide nanobundles," Applied Catalysis B, vol. 80, no. 1-2, pp. 32-41, 2008.

[16] H. Yan, R. He, J. Johnson, M. Law, R. J. Saykally, and P. Yang, "Dendritic nanowire ultraviolet laser array," Journal of the American Chemical Society, vol. 125, no. 16, pp. 4728-4729, 2003.

[17] Y. Y. Tay, S. Li, F. Boey, Y. H. Cheng, and M. H. Liang, "Growth mechanism of spherical $\mathrm{ZnO}$ nanostructures synthesized via colloid chemistry," Physica B, vol. 394, no. 2, pp. 372-376, 2007.

[18] Z. R. Tian, J. A. Voigt, J. Liu, B. Mckenzie, and M. J. Mcdermott, "Biomimetic arrays of oriented helical $\mathrm{ZnO}$ nanorods and columns," Journal of the American Chemical Society, vol. 124, no. 44, pp. 12954-12955, 2002.

[19] S. A. Morin, F. F. Amos, and S. Jin, "Biomimetic assembly of zinc oxide nanorods onto flexible polymers," Journal of the American Chemical Society, vol. 129, no. 45, pp. 13776-13777, 2007.

[20] L. P. Bauermann, A. D. Campo, J. Bill, and F. Aldinger, "Heterogeneous nucleation of $\mathrm{ZnO}$ using gelatin as the organic matrix," Chemistry of Materials, vol. 18, no. 8, pp. 2016-2020, 2006.

[21] Q. Dong, H. Su, J. Xu, D. Zhang, and R. Wang, "Synthesis of biomorphic $\mathrm{ZnO}$ interwoven microfibers using eggshell membrane as the biotemplate," Materials Letters, vol. 61, no. 13, pp. 2714-2717, 2007.

[22] S. Kaluza, M. K. Schröter, R. N. D’Alnoncourt, T. Reinecke, and M. Muhler, "High surface area $\mathrm{ZnO}$ nanoparticles via a novel continuous precipitation route," Advanced Functional Materials, vol. 18, no. 22, pp. 3670-3677, 2008.

[23] S. Busch, H. Dolhaine, A. DuChesne, et al., "Biomimetic morphogenesis of fluorapatite-gelatin composites: fractal growth, the question of intrinsic electric fields, core/shell assemblies, hollow spheres and reorganization of denatured collagen," European Journal of Inorganic Chemistry, vol. 10, pp. 16431653, 1999.

[24] A. Bigi, E. Boanini, S. Panzavolta, and N. Roveri, "Biomimetic growth of hydroxyapatite on gelatin films doped with sodium polyacrylate," Biomacromolecules, vol. 1, no. 4, pp. 752-756, 2000.

[25] M. A. Martins, C. Santos, M. M. Almeida, and M. E. V. Costa, "Hydroxyapatite micro- and nanoparticles: nucleation and growth mechanisms in the presence of citrate species," Journal of Colloid and Interface Science, vol. 318, no. 2, pp. 210-216, 2008. 
[26] H. Cölfen and M. Antonietti, "Mesocrystals: inorganic superstructures made by highly parallel crystallization and controlled alignment," Angewandte Chemie International Edition, vol. 44, no. 35, pp. 5576-5591, 2005.

[27] S. Teng, J. Shi, and L. Chen, "Formation of calcium phosphates in gelatin with a novel diffusion system," Colloids and Surfaces B, vol. 49, no. 1, pp. 87-92, 2006.

[28] J. Li, Y. Chen, Y. Yin, F. Yao, and K. Yao, "Modulation of nanohydroxyapatite size via formation on chitosan-gelatin network film in situ," Biomaterials, vol. 28, no. 5, pp. 781-790, 2007.

[29] Y. Zhai, F. Z. Cui, and Y. Wang, "Formation of nanohydroxyapatite on recombinant human-like collagen fibrils," Current Applied Physics, vol. 5, no. 5, pp. 429-432, 2005.

[30] Y. Q. Chen, J. Jiang, Z. Y. He, Y. Su, D. Cai, and L. Chen, "Growth mechanism and characterization of $\mathrm{ZnO}$ microbelts and self-assembled microcombs," Materials Letters, vol. 59, no. 26, pp. 3280-3283, 2005.

[31] D. W. James and L. Rintoul, "Protein-water Interactions in solution: the water-gelatin-electrolyte system," Australian Journal of Chemistry, vol. 35, pp. 1157-1163, 1982.

[32] Y. C. Zhang, X. Wu, X. Y. Hu, and R. Guo, "Low-temperature synthesis of nanocrystalline $\mathrm{ZnO}$ by thermal decomposition of a "green" single-source inorganic precursor in air," Journal of Crystal Growth, vol. 280, no. 1-2, pp. 250-254, 2005. 

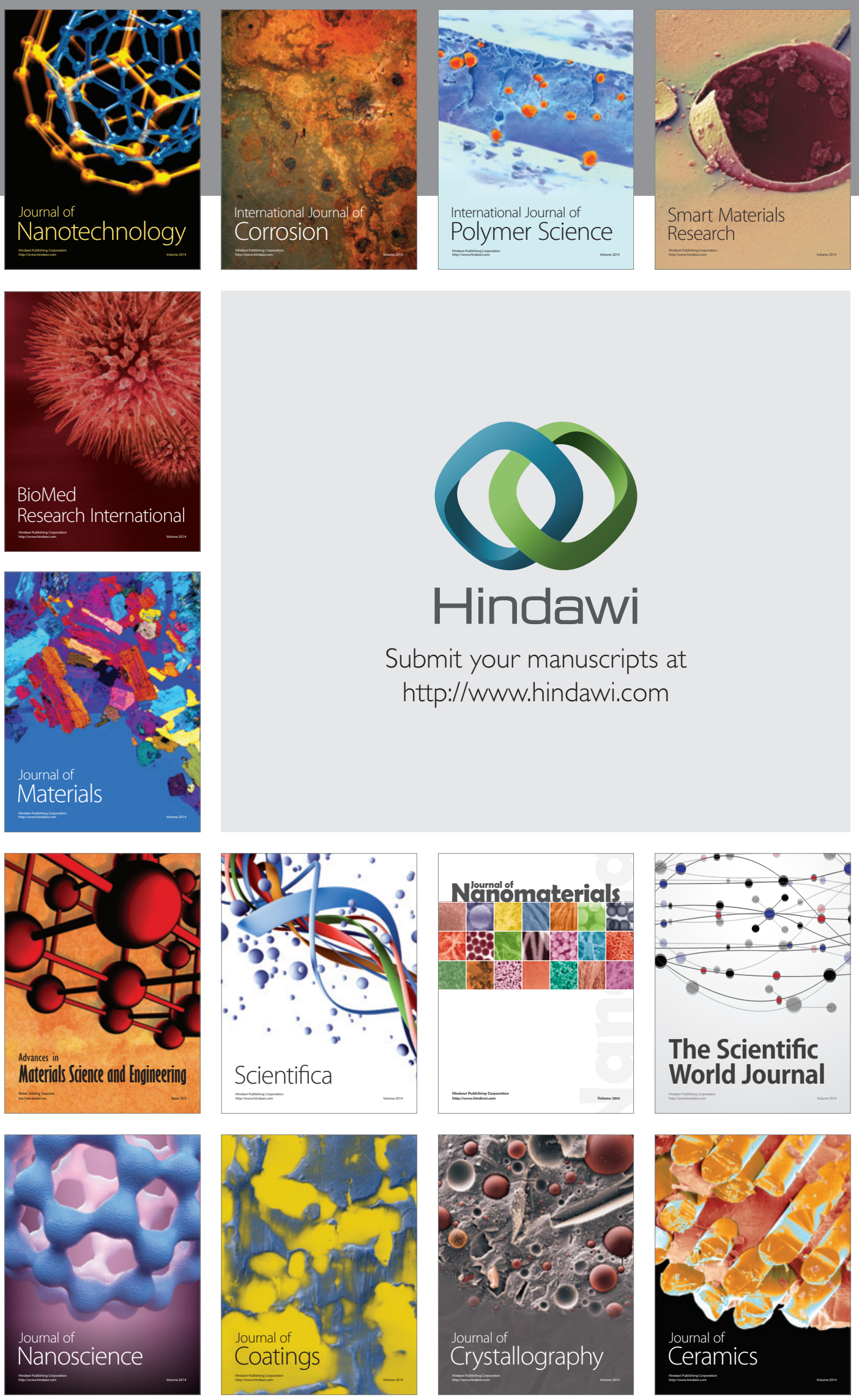

The Scientific World Journal

Submit your manuscripts at

http://www.hindawi.com

\section{World Journal}

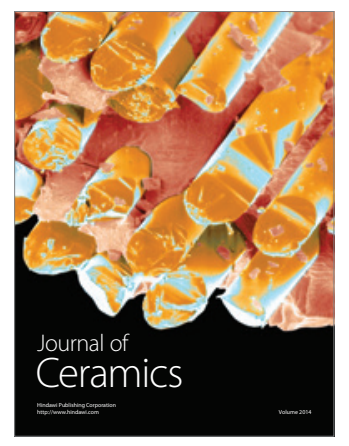

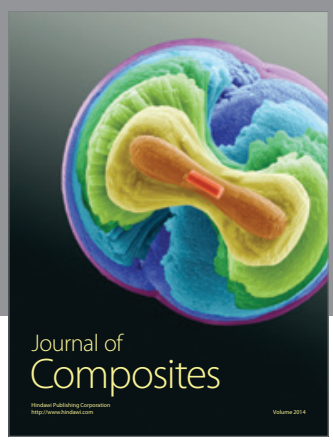
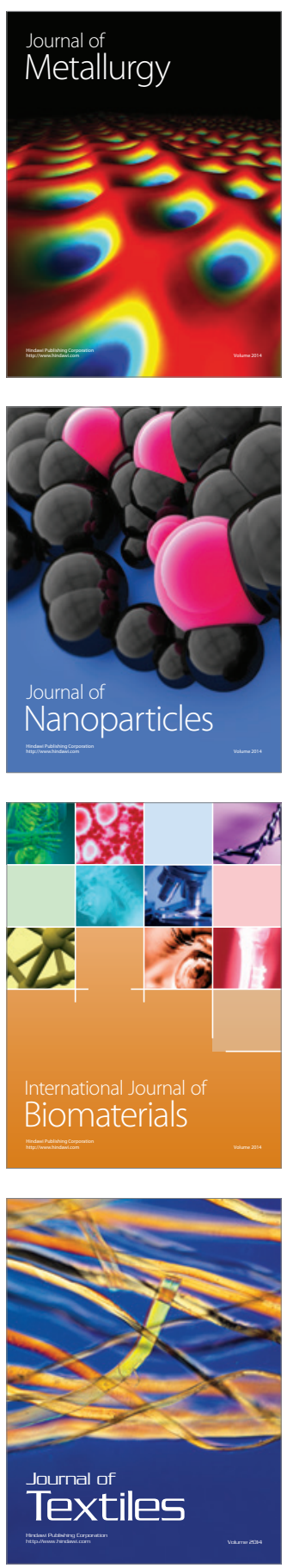